\title{
Dispositivo vestível para medição da iluminância pupilar
}

Wearable device for measuring pupilar illuminance

\author{
Maíra Vieira Dias \\ Ali Motamed \\ Paulo Sergio Scarazzato \\ J ean-Louis Scartezzini
}

\section{Resumo}

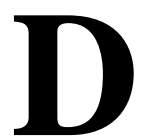

ados de exposição do olho humano à luz podem auxiliar pesquisadores e profissionais de projeto a entenderem melhor o desempenho de edifícios, relativamente ao bem-estar e saúde de seus usuários. Entretanto, as tecnologias de detecção existentes para avaliar condições internas de iluminação a partir de um ponto de vista fixo e medir as quantidades luminosas não realizam um mapeamento das cenas visuais de modo semelhante ao olho humano. Visando contribuir com a superação desta lacuna, este artigo apresenta o "OcuLux", dispositivo vestível desenvolvido no Brasil, destinado a monitorar de modo contínuo a iluminância pupilar real em ambientes internos. O "OcuLux" foi calibrado e usado durante 7 dias em novembro de 2016 no Solar Energy and Building Physics Laboratory (LESO-PB) no campus da École Polytechnique Fédérale de Lausanne (EPFL) em Lausanne (Suíça), sob céus parcialmente encoberto e encoberto para diagnosticar a dose de luz recebida por pessoa exercendo atividades típicas de escritório. Os resultados mostraram que não foi encontrada diferença significativa entre a dose de luz recebida pela manhã e durante a tarde. Embora o "OcuLux" apresente faixa de medição limitada, seu uso em uma situação real indicou que o mesmo é confiável para medições da iluminância pupilar.

Palavras-chave: Conforto visual. Dispositivo de medição. Dose de luz. Iluminância pupilar. Saúde.

${ }^{1}$ Maíra Vieira Dias ${ }^{1}$ Universidade Estadual de Campinas Campinas - SP - Brasil

${ }^{2}$ Ali Motamed ¿École Polytechnique Fédérale de Lausanne Lausanne - Switzerland

${ }^{3}$ Paulo Sergio Scarazzato ${ }^{3}$ Universidade Estadual de Campinas Campinas - SP - Brasil

${ }^{4} J$ ean-Louis Scartezzini 4École Polytechnique Fédérale de Lausanne Lausanne - Switzerland

Recebido em 18/04/18 Aceito em 21/07/18

\section{Abstract}

Data on the exposure of the human eye to light can help researchers and design professionals to better understand building performance with regards to the wellbeing and health of users. However, technologies currently used to evaluate indoor lighting conditions from a fixed point of view and measure luminous quantities are unable to map out visual scenes in a similar way to the human eye. With the aim of contributing to overcome this gap, this paper presents "OcuLux", a wearable device made in Brazil, designed to continuously monitor pupilar illuminance for indoor environments. "OcuLux" was calibrated and the sensor was worn for 7 days in November 2016 at the Solar Energy and Building Physics Laboratory (LESO-PB) at École Polytechnique Fédérale de Lausanne (EPFL) Lausanne campus (Switzerland), under partly cloudy and overcast skies to diagnose the light dose received by a person performing typical office activities. The results showed that no significant differences were found between the light dose received in the morning and in the afternoon. Although "OcuLux" has a limited measuring range, its use in a real situation has indicated that it is reliable for measurements of pupilar illuminance.

Keywords: Visual comfort. Measurement device. Light dose. Pupilar illuminance. Health. 


\section{Introdução}

A luz que penetra no olho humano não é responsável apenas pelo processo de formação de imagens que promovem a visão, mas também por vários efeitos biológicos não visuais (também conhecidos como não formadores de imagens) que provocam $\mathrm{o}$ atraso do relógio biológico $\mathrm{e}$ influenciam o estado de alerta, a cognição e o humor.

A configuração dos ambientes internos, incluindo sua iluminação, pode ter grande impacto sobre o comportamento dos usuários. $\mathrm{O}$ ambiente de trabalho, na maioria das vezes configurado como um escritório, tem modificado sua natureza ao longo dos anos em decorrência de mudanças na forma como o trabalho é realizado. A introdução dos computadores no ambiente de trabalho foi o marco nessa mudança, que continua devido às tecnologias emergentes (BOYCE, 2014).

Segundo Boyce e Raynahn (2009), a função da iluminação no ambiente de trabalho é principalmente tornar as informações visíveis, sem desconforto. Consequentemente, a mudança do trabalho baseado em leituras e escritas em documentos de papel para o trabalho realizado na tela do computador tem implicações importantes para a iluminação. No primeiro caso, a superfície principal a ser vista é horizontal e o aumento da quantidade de luz torna qualquer informação nessa superfície mais visível. No segundo caso, a superfície de trabalho é vertical e o aumento da quantidade de luz torna a informação exibida na tela menos visível. Como a maioria dos ambientes de trabalho apresenta a combinação dos dois tipos de tarefa, os sistemas de iluminação projetados devem ser satisfatórios para materiais/superfícies que são autoiluminadas (como a tela de computadores) e superfícies a serem vistas através da luz refletida (como papéis). Deve-se ainda considerar os campos de visão possíveis no ambiente.

As recomendações para iluminação em ambientes de trabalho geralmente são baseadas no critério visual, no qual a iluminância no plano horizontal tem sido o principal parâmetro de projeto ${ }^{1}$ (ARIES, 2005). A norma europeia EN 12464-1 Light and lighting. Lighting of work places - Part 1: Indoor work places (EUROPEAN..., 2011) recomenda uma iluminância horizontal de 300 a 500 lx para as atividades a serem desenvolvidas em um escritório tradicional.

${ }^{1}$ Tais padrões são baseados em escritórios tradicionais onde as atividades mais comuns são desenvolvidas envolvendo escrita e leitura de papéis sobre as mesas.
Considerando que os efeitos biológicos não visuais da iluminação dependem da quantidade de luz, distribuição do espectro, distribuição espacial, tempo e duração da exposição, não é suficiente basear-se apenas na iluminância nos planos horizontal e vertical para desenvolver um projeto de iluminação.

Para otimizar os efeitos biológicos não visuais, níveis de iluminação maiores que os requeridos não são necessários durante todo o dia. Conforme a DIN SPEC 67000 Biologically effective illumination. Design guidelines (DEUTSCHES..., 2013) a iluminância vertical mínima recomendada ao nível dos olhos é de 250 lx com uma Temperatura de Cor Correlacionada (CCT) de $8.000 \mathrm{~K}$ pela manhã (8h10h) e no início da tarde (13h-14h), 200 lx com CCT de $3.000 \mathrm{~K}$ durante o intervalo do almoço (12h-13h) e à noite (18h-20h), enquanto que durante o resto do dia a iluminância vertical deve corresponder às exigências da tarefa visual.

Kaida et al. (2006) demonstraram, sob condições controladas, que 30 minutos de exposição à luz natural brilhante próxima a janelas (com iluminância pupilar entre 1.000 a 4.000 lx) foi quase tão eficaz quanto um cochilo curto na redução da sonolência normal pós almoço em sujeitos normais. Trabalhos realizados em condições laboratoriais mostraram que o marca-passo circadiano muda de fase em resposta a níveis relativamente baixos de uma fonte de luz branca de espectro de banda larga ( 100 lx no plano da córnea ou 300-500 lx no plano horizontal). Mais adiante foi descoberto que os níveis de radiação óptica entre 50-100 lx na córnea poderiam afetar o sistema circadiano dos seres humanos (ILLUMINATING..., 2008). Cajochen (2015) constatou que luz enriquecida de azul na faixa de 40 lx e proveniente de LED - como retroiluminação de telas de computadores à noite são fortes o suficiente para estimular respostas de alerta significativas. Foi ainda determinado que a exposição noturna durante duas horas sob as condições de iluminação citadas aumenta o desempenho em diferentes domínios cognitivos e afeta negativamente a qualidade e a duração do sono.

Níveis de radiação óptica requeridos para impactar o relógio biológico em ambientes não laboratoriais ainda são desconhecidos. Entretanto, estudo desenvolvido por Burgess e Eastman (2004) usando o dispositivo Actiwatch- $L^{2}$ (Figura 1) em volta do pescoço de sujeitos para medir a radiação óptica

20 Actiwatch-L é um dispositivo compacto que possui um acelerômetro multidirecional para monitorar o grau e a intensidade dos movimentos com várias amostragens por segundo e geralmente é usado no pulso. 
sugeriu que níveis em torno de 40 lx medidos em uma superfície vertical (ou seja, aproximadamente 200 lx no plano horizontal) por três horas à noite (entre 22h00-01h00) pode induzir a um atraso de fase no marca-passo circadiano. Em estudo de campo, Münch et al. (2012) verificaram que uma exposição à luz natural por 6 horas $(\sim 1.000 \mathrm{~lx}$ ao nível dos olhos) começando 4 horas após acordar por dois dias consecutivos aumentou o desempenho noturno no segundo dia de experimento quando comparado com uma exposição a 176 lx no plano da córnea com uma fonte de luz de $3.700 \mathrm{~K}$.

Em vista do exposto, uma avaliação adequada do fluxo de luz que atinge o olho humano em ambientes internos é um passo crucial para caracterizar e mitigar questões como privação do sono, depressão, redução da produtividade e do estado de alerta. Todavia, como relatam Vandahl et al. (2011), apenas a iluminância vertical próxima ao olho vem sendo medida, enquanto o campo de visão e o movimento da cabeça são ignorados. As tecnologias de detecção existentes (por exemplo Actiwatch-L, Daysimeter e Dimesimeter) avaliam as condições de iluminação em um ambiente interno, contudo não realizam um mapeamento das cenas visuais de modo semelhante ao olho humano.

Mesmo o Actiwatch-L (Bend, OR, EUA) (Figura 1) equipado com um fotodiodo de bordo em miniatura para medição da quantidade e duração da iluminância, quando usado no pulso não fornece uma leitura precisa da luz incidente no plano das córneas. Outra desvantagem é a interpretação da ausência de movimentos como período de sono, comprometendo estudos que visem a caracterização da insônia. O Daysimeter (Lighting Research Center - LRC, NY, EUA) (Figura 2), opera apenas no plano de uma das córneas e as pesquisas envolvendo o dispositivo buscam determinar como a luz afeta o sistema circadiano. O Dimesimeter (Figura 3), também desenvolvido pelo LRC, pode ser usado em vários locais do corpo e quando fixado em armações de óculos registra a radiação óptica incidente próxima aos olhos. Todavia, estudos apontam que quando colocado em outras partes do corpo apresenta diferenças nas quantidades absolutas no registro da luz (DIAS et al., 2015).

Pesquisas voltadas para a compreensão de como a luz influencia o ciclo circadiano utilizando o Daysimeter e o Dimesimeter encontram-se em estágio avançado. Em contrapartida, a carência no entendimento do quanto a luz pode impactar o sistema visual humano levou à criação do "LuxBlick" (Figura 4) por Hubalek; Zoschg; Schierz (2006) na Eidgenössische Technische Hochschule Zürich (ETH Zürich, Suíça). As pesquisas tiveram continuidade na Technischen Universität Ilmenau (TU Ilmenau, Alemanha) e levaram a um desenvolvimento adicional do sistema original (DIAS et al., 2015). O "LuxBlick" apresenta dois sensores fotométricos fixados em armações de óculos e tem como objetivo fornecer dados estatísticos das iluminâncias expostas nos locais de trabalho e as distribuições de frequências dos movimentos nos olhos associados com as distribuições de luminâncias no ambiente (HUBALEK; SCHIERZ, 2005).

Derivado do dispositivo alemão "LuxBlick", o dispositivo vestível brasileiro "OcuLux" foi desenvolvido e fornece continuamente a iluminância pupilar real (lux). Diferentemente daquele que lhe deu origem e que possui um sensor para registro da iluminância tradicional baseada na curva de sensibilidade fotópica e outro para registro da luz azul, o “OcuLux” conta com um sensor RGB. Este artigo apresenta o dispositivo em questão e seu processo de calibração, assim como os resultados obtidos em experimento de campo preliminar para diagnosticar a quantidade de luz (aspectos visuais) que atinge o olho humano durante o trabalho. Foi ainda calculada a dose de luz recebida pelo usuário em uma sala no Solar Energy and Building Physics Laboratory (LESO-PB) da École Polytechnique Fédérale de Lausanne (EPFL) em Lausanne (Suíça).

Figura 1 - Actiwatch-L

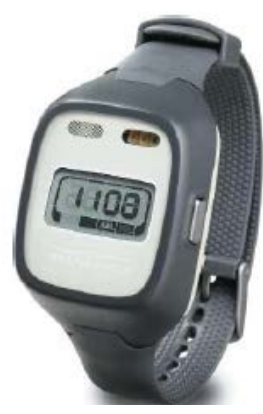

Fonte: Philips Respironics (2011). 
Figura 2 - Daysimeter

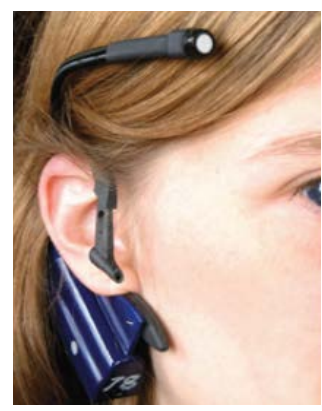

Fonte: Jernigan (2009).

Figura 3 - Dimesimeter

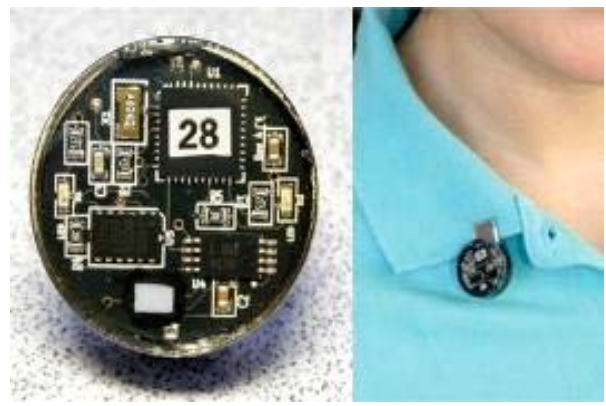

Fonte: Lighting Research Center (2013).

Figura 4 - LuxBlick

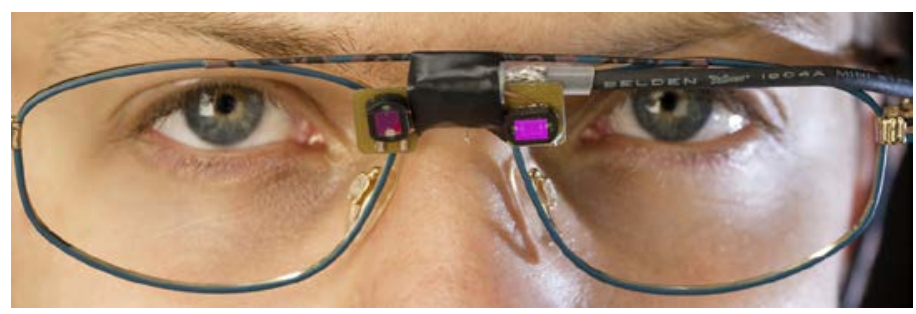

Fonte: Vandahl et al. (2011).

\section{Procedimentos metodológicos}

Os procedimentos metodológicos utilizados foram divididos em seis etapas:

(a) seleção do ambiente de trabalho e caracterização do clima em Lausanne;

(b) caracterização do sujeito da pesquisa;

(c) caracterização do sistema de iluminação;

(d) aferição dos níveis de iluminância através do "OcuLux";

(e) estudo experimental; e

(f) análise dos resultados.

\section{Seleção do ambiente de trabalho}

O experimento ocorreu no LESO-PB no campus da EPFL em Lausanne (Suíça). Lausanne está situada na parte francófona da Suíça (latitude 42³2'N, longitude $\left.6^{\circ} 36^{\prime} \mathrm{E}\right)$ e é a capital do cantão de Vaud. A $495 \mathrm{~m}$ de altitude, a cidade está localizada a $62 \mathrm{~km}$ a noroeste de Genebra. Apresenta clima quente e temperado e as temperaturas médias estão entre $3{ }^{\circ} \mathrm{C}$ e $20{ }^{\circ} \mathrm{C}$, enquanto extremos podem atingir $-10{ }^{\circ} \mathrm{C}$ e $37{ }^{\circ} \mathrm{C}$. As chuvas são uniformemente distribuídas ao longo do ano (LAUSANNE, 2017).

Para realização do estudo experimental foi selecionada a sala LE001 no pavimento térreo do LESO-PB e voltada para a face sul (Figura 5a). A sala possui $15,7 \mathrm{~m}^{2}$ de área e $2,8 \mathrm{~m}$ de pé-direito. A 
sala conta com mobiliário padrão e duas estações de trabalho principais (Figura 5b).

A escolha do ambiente deve-se ao fato do mesmo apresentar dois sensores instalados, capazes de medir variáveis fotométricas e índices de ofuscamento (Figura 5c). O primeiro sensor, usualmente fixado em um tripé e na altura do campo visual do usuário, está localizado ao lado da estação de trabalho posicionada com a janela à sua esquerda e monitora as principais variáveis fotométricas (como iluminância pupilar e luminância média) e índices de ofuscamento (como Daylight Glare Index - DGI e Daylight Glare Probability - DGP). $\mathrm{O}$ segundo sensor encontra-se fixado no teto e é utilizado para garantir a iluminância horizontal necessária no plano de trabalho $\left(\mathrm{E}_{\mathrm{h}}\right)$ de acordo com a EN 12464-1 (EUROPEAN..., 2011). Há ainda um detector de presença que monitora a ocupação da sala para ativação dos sistemas de iluminação artificial e sombreamento.

Pesquisa em andamento no LESO-PB utilizando o dispositivo vestível está investigando a localização ideal para o primeiro sensor a fim de minimizar as discrepâncias entre a iluminância vertical registrada em um ponto fixo e as quantidades registradas em pelo dispositivo vestível. Assim, para o presente experimento o primeiro sensor foi fixado no computador (VDT) (Figura 6).

Figura 5 - (a) Fachada Sul do LESO-PB. Em destaque a sala LE001 onde o experimento foi realizado. (b) Interior da sala. (c) Localização usual dos sensores

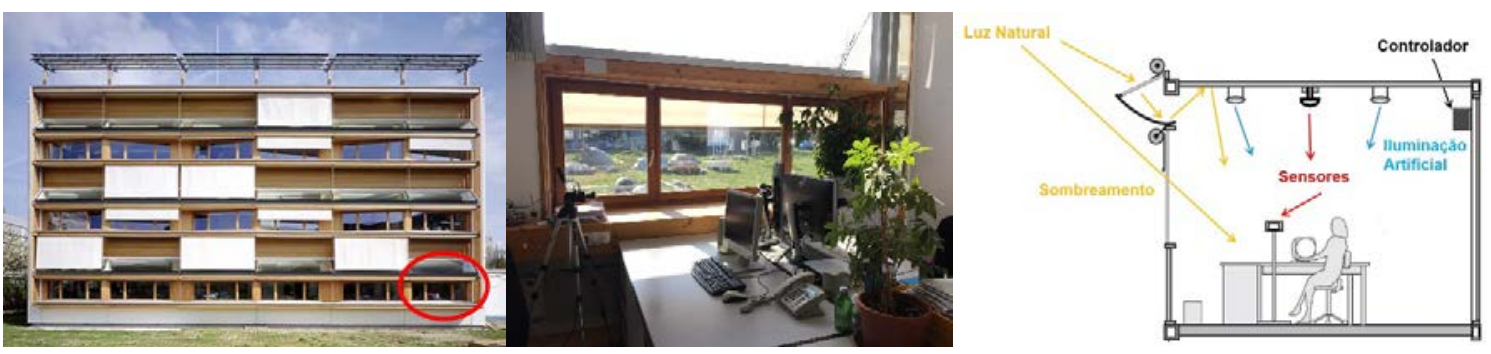

Figura 6 - Sensor fixado no computador (VDT)

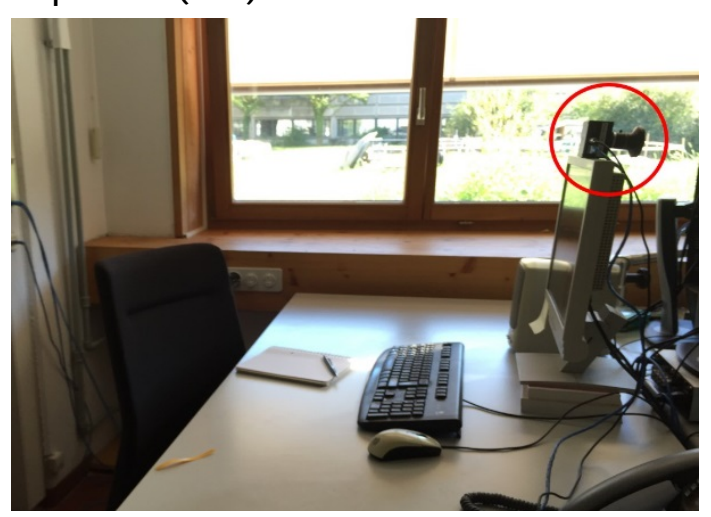

\section{Caracterização do sujeito da pesquisa}

Devido ao posicionamento dos sensores e controladores manuais na LE001, foi determinado que o sujeito da pesquisa ocuparia a estação de trabalho posicionada com a janela à sua esquerda (Figura 7a e 7b). O sujeito selecionado foi um estudante de pós-graduação, brasileiro, 35 anos, que estava trabalhando permanentemente na estação acima descrita e apresentava horário regular de turno de trabalho (08-12h e 13-17h). O sujeito possuía visão normal (sem lentes corretivas) e manteve um registro diário com informações sobre as medições.

\section{Caracterização do sistema de iluminação}

Para captação da luz natural, o edifício referido apresenta além das janelas convencionais, um Anidolic Daylighting System (ADS) integrado à fachada sul (Figuras 8a a 8c). Esta fachada é composta por 18 ADS distintos (sendo 6 por pavimento) que iluminam 14 salas, 01 sala de seminários e 01 oficina. Por bloquear parte do componente direto do fluxo de luz natural incidente, o ADS contribui para reduzir a iluminância no plano de trabalho e as luminâncias de objetos e paredes do local. O fluxo de luz natural bloqueado é redirecionado para o teto e distribuído de modo 
uniforme no interior da sala. O sistema de iluminação artificial complementar é caracterizado por LEDs com $23 \mathrm{~W}$ de consumo e fluxo luminoso de $2.400 \mathrm{~lm}$, CCT de $4.000 \mathrm{~K}$, Índice de Reprodução de Cor (CRI) superior a 80. É ainda esperado que o Índice de Ofuscamento Unificado (UGR) detectado pelos usuários seja inferior a 19 (MOTAMED; DESCHAMPS; SCARTEZZINI, 2017).
O sistema de sombreamento é composto por dois painéis externos motorizados, em material têxtil translúcido. O painel superior cobre o ADS e é usado para controle do ofuscamento. O painel inferior cobre a janela convencional e é usado para proteção térmica (Figura 9). Os painéis podem ser controlados tanto através de quatro interruptores manuais no interior da sala (um para descer e outro para subir para cada painel) (Figura 7b) ou através de uma rede de comunicação $\mathrm{KNX}^{3}$.

Figura 7 - (a) Posição ocupada pelo usuário durante o experimento. (b) Localização dos controles manuais e da iluminação artificial

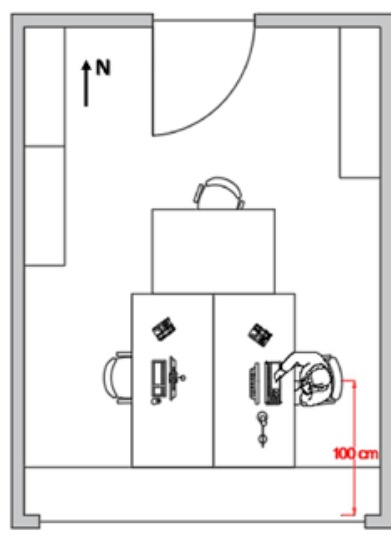

painel externo motorizado

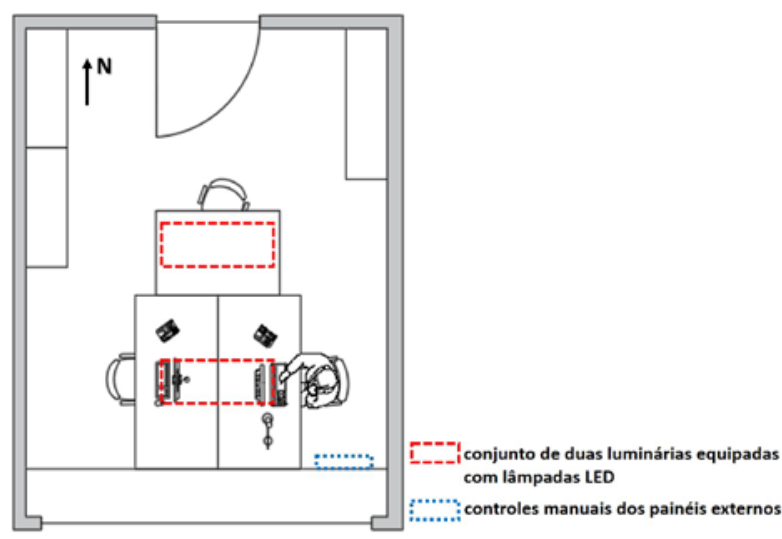

painel externo motorizado

Figura 8 - (a) ADS na fachada sul; (b) Seção transversal na fachada sul; e (c) Vista esquemática da sala LE001 equipada com ADS

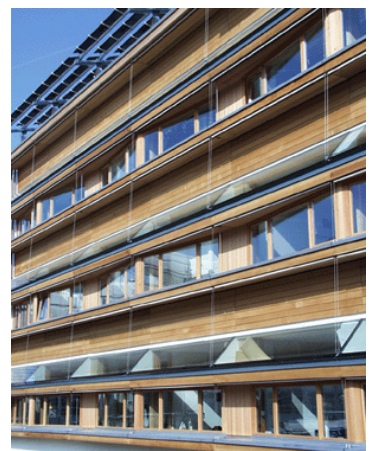

(a)

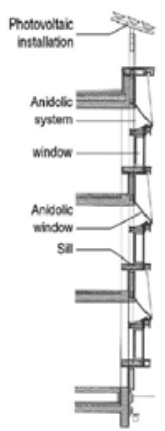

(b)

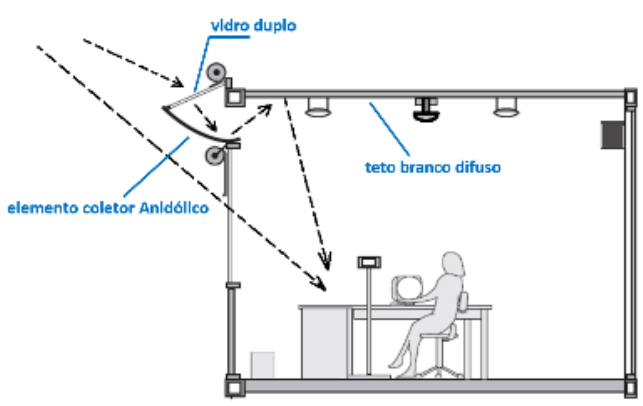

(c)

Fonte: (a) Solar Energy and Building Physics Laboratory (2016); e (b) Altherr e Gay (2002).

3Uma rede de comunicação KNX permite que vários elementos que compõem um sistema se comuniquem entre si numa rede complexa de troca de mensagens, em uma linguagem única. 0 sistema efetua a comunicação direta entre todos os elementos de forma simples, sem recorrer a uma hierarquia ou a um dispositivo central de controle. Assim, cada elemento é reconhecido de forma independente. Os sensores detectam e enviam mensagens para que o sistema possa atuar de acordo com sua programação. 
Figura 9 - Painéis externos motorizados

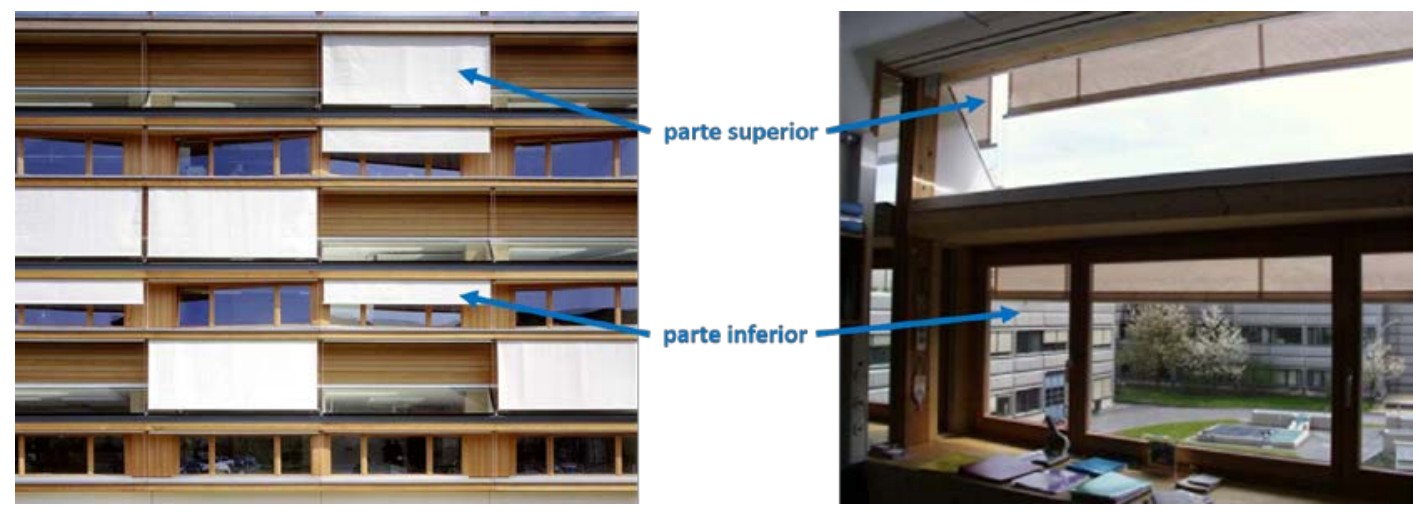

Fonte: adaptado de Linhart (2010).

O sistema de iluminação, os sensores e os painéis externos motorizados se comunicam e são controlados através de uma plataforma de controle ad-hoc que garante a aquisição e registro dos dados, inicialização correta e atuação dos comandos (LINHART, 2010; MOTAMED; DESCHAMPS; SCARTEZZINI, 2016). A plataforma considera o DGP, a E $E_{h}$ e o perfil solar para determinar a posição relativa dos sombreamentos, bem como a quantidade de luz solar disponível para ativação da iluminação artificial. A sala LE001 possui um controlador que ativa o sistema de sombreamento quando os índices de DGP registrados são superiores a 5\%. O sistema de iluminação artificial complementar é ativado quando a $E_{h}$ no plano de trabalho é inferior a 200 lx e desativado quando superior a 700 lx. Segundo Wienold e Christoffersen (2006), o limite recomendado como ofuscamento imperceptível do DGP deve ser 35\%. Por sua vez, a EN 12464-1 (EUROPEAN..., 2011) estabelece uma $\mathrm{E}_{\mathrm{h}}$ mínima de 300 lx no plano de trabalho. Os valores determinados para o controlador foram determinados em decorrência de outros experimentos relacionados ao desempenho visual que ocorrem na LE001.

\section{Aferição dos níveis de iluminância através do "OcuLux"}

\section{Dispositivo "OcuLux"}

Para investigar o fluxo de luz que atinge o olho dos usuários durante sua jornada de trabalho, o dispositivo vestível brasileiro "OcuLux" foi desenvolvido em uma plataforma Arduino.

A plataforma Arduino é uma plataforma de prototipagem eletrônica open-source apoiada em hardware e software flexíveis. O hardware contém a placa de prototipagem (protoboard), na qual diversos sensores são conectados a um microcontrolador que permite a sua manipulação por meio de scripts de programação, estabelecendo um fluxo de dados a partir das conexões serial ou remota (ARDUINO, [201-]).

O “OcuLux” possui essencialmente duas partes: um data logger que contém o circuito eletrônico e óculos de segurança do trabalho com um sensor RGB (Red, Green, Blue) sensível às regiões vermelha $(\lambda=615 \mathrm{~nm})$, verde $(\lambda=540 \mathrm{~nm})$ e azul $(\lambda=465 \mathrm{~nm})$ do espectro para registro do fluxo de luz (Figura 10). O dispositivo é capaz de realizar registros com uma resolução temporal de $01 \mathrm{~s}$ e salvar esses registros continuamente.

A Figura 11 mostra um diagrama de blocos do circuito eletrônico. O bloco central é o Arduino $\mathrm{NANO}^{4}$ que é conectado ao relógio interno - Real Time Clock (RTC) e ao módulo Secure Digital (SD). Todo o conjunto é alimentado por uma bateria de $9 \mathrm{~V}$. O RTC registra a hora absoluta para os dados coletados e gera a cada segundo um pulso que aciona uma nova medição. É alimentado por uma bateria de lítio CR 2032 de $3 \mathrm{~V}$ que permite a gravação em um determinado período de tempo e mantém a frequência independente da bateria de 9 V. Assim, é possível substituir a bateria durante um experimento sem comprometer as configurações do RTC e o processo de registro de dados. A bateria de $9 \mathrm{~V}$ permite até cerca de 20 horas de medições. Como o Arduino possui apenas $2 \mathrm{~K}$ de memória permanente e esta não é suficiente para armazenar os dados, utilizou-se uma memória interna através de um módulo SD. 
Figura 10 - Dispositivo vestível "OcuLux" que permite avaliar a iluminância pupilar (Iux) em tempo real

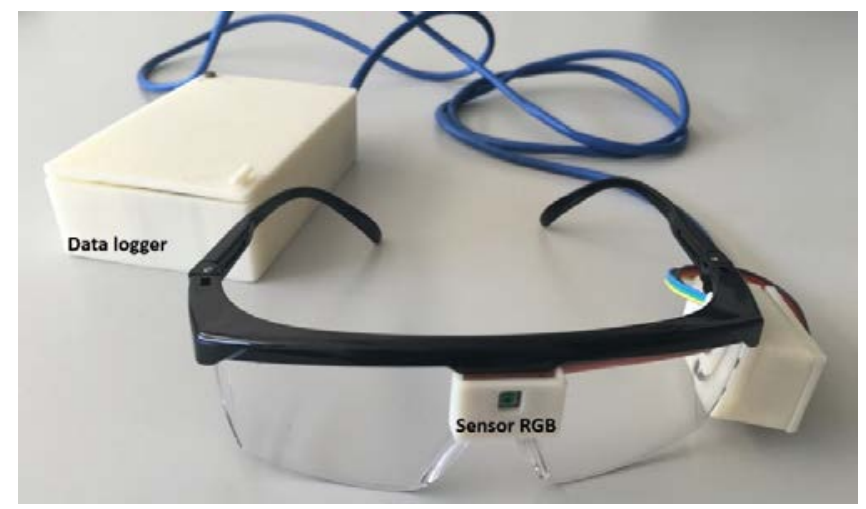

Figura 11 - Diagrama eletrônico do "OcuLux"

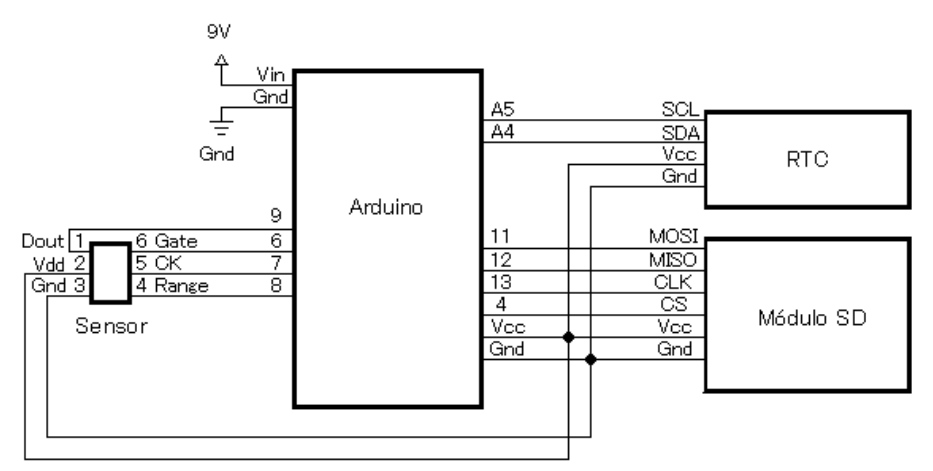

O sensor RGB S9706 (Hamamatsu Photonics, Tóquio, Japão) - fixado nos óculos de segurança do trabalho e fora do campo de visão - se comunica com o Arduino, o RTC e o módulo SD através de um cabo Ethernet. O sensor converte o fluxo de luz incidente em sinais digitais de 12-bits. Um amplificador é usado para cada elemento do fotodiodo RGB disposto em um padrão mosaico, permitindo medições precisas dos componentes RGB do fluxo de luz incidente. Por possuir um conversor analógico para digital (A/D), há uma redução no número de erros durante as medições devido à influência de ruídos durante a troca de sinais e informações entre o Arduino e o sensor (HAMAMATSU..., 2016).

Por ser projetado para medições internas, optou-se pela configuração de alta sensibilidade do fotodiodo por esta cobrir uma área fotossensível eficaz de 9x9 elementos do fotodiodo de silício no padrão mosaico.

Para evitar uma saturação do sensor, foi determinado o tempo de integração do mesmo. O tempo de integração corresponde ao período em que o detector está coletando a luz incidente e é uma variável que deve ser previamente definida pelo usuário e que pode ser modificada através do software utilizado. Os níveis de luz incidente e o tempo de integração determinam o número de elétrons coletados em cada pixel. Se a luz incidente gera mais elétrons que o pixel pode suportar, o pixel fica saturado. $\mathrm{O}$ aumento do tempo de integração aumenta o número de contagens em todo o espectro, podendo, portanto, haver alguma região que fique saturada quando o tempo de integração é aumentado. Para o sensor RGB S9706 esse tempo varia na faixa de centenas de milissegundos.

Desta forma, foram realizados testes utilizando o “OcuLux" na sala LE001 com diferentes tempos de integração (20 ms, 30 ms, 50 ms, 80 ms, 100 ms). Os dados obtidos mostraram um melhor desempenho do dispositivo com um tempo de integração equivalente a $30 \mathrm{~ms}$, que permite registros sem saturação das amostras até 3.500 lx.

Para permitir a interface entre o usuário e o dispositivo foi desenvolvido um software. O processo de configuração dessa interface é simples, rápido e intuitivo. A cada nova medição, a data, o tempo de medição e o tempo de integração devem ser definidos. Ao término das medições os dados podem ser acessados pelos usuários por meio da conexão do dispositivo a um computador através de um cabo USB. Para evitar perdas, os dados só são deletados após a inserção do comando delete. Caso isto não ocorra, os dados das medições são armazenados continuamente na memória do dispositivo. 


\section{Calibração}

Para verificar a linearidade do “OcuLux", o dispositivo foi testado por mais de 40 horas sob diferentes condições de iluminação natural e artificial, na Universidade Estadual de Campinas (UNICAMP) e no LESO-PB no campus da EPFL. Durante os testes no LESO-PB, os óculos de segurança do trabalho foram fixados em um tripé juntamente com um luxímetro de referência Chroma Meter CL-200A Konica Minolta (Konica Minolta, Tóquio, Japão), de modo que ambos os sensores ficassem dispostos lado a lado.

Como apresentado por Dias et al. (2017), para melhorar a precisão das medições, o processo de calibração foi realizado sob condições internas de luz natural (para céus claro e encoberto) no LESOPB e sem a interferência da incidência da luz solar direta. Tal processo teve como objetivo a calibração para aferição dos efeitos visuais da luz. Apesar de ser possível uma calibração para aferição dos efeitos biológicos não visuais, o sensor não foi preparado e utilizado para este fim no presente experimento, visto que tais medições fugiam ao escopo da pesquisa.

Os dados coletados pelo "OcuLux" foram comparados com aqueles registrados pelo luxímetro de referência. A saída digital do sensor $\left(\mathrm{RGB}_{\text {raw }}\right)$ varia de 0 a 4095 dígitos. Utilizando os parâmetros de fotossensibilidade fornecidos pelo sensor $(S r=5,8, \quad S g=4,1, \quad S b=1,9)$, a saída digital foi traduzida para valores lineares $\left(R G B_{\text {linear }}\right)$ (Equação 1):

$R G B_{\text {linear }}=\left(R_{\text {raw }} * 5,8+G_{\text {raw }} * 4,1+B_{\text {raw }} * 1,9\right) \quad$ Eq. 1

Onde:

$R G B_{\text {linear }}=$ valores lineares obtidos para o sensor;

$R_{\text {raw }}=$ saída digital do canal R;

$G_{\text {raw }}=$ saída digital do canal G; e

$B_{\text {raw }}=$ saída digital do canal B.

Na sequência, foi usada a equação fornecida por Jacobs (2014) para conversão dos dados em RGB para lux, onde 179 é a eficiência luminosa padrão assumida (Equação 2):

$Y_{\text {rel }}=179 *\left(R_{\text {linear }} * 0,265+G_{\text {linear }} * 0,67+B_{\text {linear }} * 0,065\right)$

Eq. 2

Onde:

$Y_{\text {rel }}=$ iluminância relativa;

$R_{\text {linear }}=$ valor linear do canal $\mathrm{R}$;

$G_{\text {linear }}=$ valor linear do canal G; e

$B_{\text {linear }}=$ valor linear do canal B.
Por último, os valores de iluminância relativa $\left(Y_{\text {rel }}\right)$ foram transformados usando um fator de conversão obtido através dos valores medidos pelo luxímetro de referência.

\section{Processamento de dados}

Como os sensores para medição das variáveis fotométricas instalados na sala LE001 estão programados para realizar coletas em intervalos de $16 \mathrm{~s}$, o mesmo intervalo foi definido para os registros do "OcuLux". Na sequência, foi selecionado o tempo de integração equivalente a $30 \mathrm{~ms}$ e as amostras que apresentaram saturação no registro foram descartadas. A amostra final foi traduzida para valores lineares (Equação 1) e os valores de iluminância relativa foram obtidos através da Equação 2 apresentada no item anterior. Os valores de iluminância foram então transformados usando o fator de conversão obtido durante a calibração. Utilizando um código no software MATLAB (2016) os dados foram agrupados a cada minuto e após tal procedimento as amostras foram analisadas.

\section{Estudo experimental}

A fim de verificar a confiabilidade do dispositivo vestível "OcuLux" como um medidor de iluminância frontal em tempo real, o estudo experimental ocorreu no período do outono, em novembro de 2016, sob condições de céus parcialmente encoberto e encoberto. $\mathrm{O}$ sujeito usou o dispositivo “OcuLux" por 7 dias de 08-12h e 1317h. Cabe ressaltar que o dispositivo vestível foi usado apenas enquanto 0 sujeito estava desempenhando suas atividades no interior da sala LE001.

Durante o experimento, os sistemas de sombreamento e de iluminação artificial foram controlados automaticamente para garantir o conforto visual do usuário (minimizando os riscos de ofuscamento) e iluminação suficiente no plano de trabalho (otimizando a iluminância no plano de trabalho). O usuário, por sua vez, teve acesso aos controles manuais no interior da sala para alterar a posição dos painéis externos motorizados e acionar a iluminação artificial de acordo com suas preferências.

\section{Análise dos resultados}

Durante o experimento o sujeito utilizou o "OcuLux" por 08h diárias e os dados coletados foram comparados àqueles registrados pelo sensor fixo instalado no VDT. Foi calculada a dose de luz por hora [lux.h] recebida. A dose de luz por hora [lux.h] foi obtida pela integração da iluminância 
medida durante o período equivalente a uma hora. Uma dose diária de luz é determinada através da soma das doses de luz por hora. Desta forma, foram determinadas as doses diárias de luz para cada dia de experimento. Em decorrência da saturação das amostras e/ou da necessidade de se ausentar do ambiente analisado, os dias com dados inferiores a 06h de coleta foram desconsiderados. Foi ainda verificado o tempo de exposição aos diferentes níveis de iluminância aos quais o usuário foi exposto durante sua jornada de trabalho.

\section{Resultados e discussões}

Este experimento de campo preliminar faz parte de uma pesquisa maior em curso no LESO-PB para investigar a localização ideal do sensor usualmente fixado no tripé. Neste experimento o sensor foi fixado no VDT e as iluminâncias registradas por esse sensor foram comparadas àquelas obtidas pelo "OcuLux".

Coletas realizadas para dias com céu encoberto mostraram uma estabilidade nos registros de ambos os sensores no período da manhã, como pôde ser observado no dia 18 de novembro. A iluminância pupilar média registrada pelo "OcuLux" (azul) foi de $90 \mathrm{~lx}$, enquanto o sensor no VDT (laranja) apresentou $\sim 30 \mathrm{~lx}$.

O aumento da exposição à luz solar no início da tarde contribuiu para um aumento no registro da iluminância pupilar (Figura 12a). Tal fato pode ser verificado pela aquisição de dados pelos sensores fixos no teto e no VDT, que permitiram o registro da $\mathrm{E}_{\mathrm{h}}$ no plano de trabalho e do DGP, assim como a posição dos painéis externos motorizados e o status da iluminação artificial complementar (Figura 12b). Após esse período ambos os sensores registraram uma queda nos níveis de iluminância.

Em relação aos painéis externos motorizados, a posição $100 \%$ indica que o sistema não foi ativado e $0 \%$ que o mesmo cobre toda a janela. Para o sistema de iluminação, a posição $100 \%$ indica que o sistema foi acionado. Como a $\mathrm{E}_{\mathrm{h}}$ (vermelho) no plano de trabalho se manteve inferior a $700 \mathrm{~lx}$ durante toda a manhã, a iluminação artificial (verde) esteve acionada. Com o aumento nos índices da $E_{h}$ entre 11h40-14h40, a iluminação artificial foi desativada e os painéis inferior (azul) e superior (amarelo) passaram a cobrir $25 \%$ da janela convencional e $90 \%$ do ADS, respectivamente.

Com o acionamento dos painéis, a $\mathrm{E}_{\mathrm{h}}$ decai e a condição de conforto visual é reestabelecida. Mesmo o DGP (roxo) registrado entre 10h10-14h20 tendo sido superior ao valor estabelecido para ativação do controlador, por estar na faixa de conforto, o usuário enviou comando para a plataforma (via comandos manuais no interior da sala) para que o painel superior permanecesse desativado. A diminuição da $E_{h}$ e do DGP promoveu o retorno dos painéis à sua posição original. Tal ação em conjunto com o acionamento da iluminação artificial contribuiu para o fornecimento de iluminação suficiente no plano de trabalho.

\section{Figura 12 - Dados para 18 de novembro de 2016 sob céu encoberto: (a) Registro das iluminâncias pelo "OcuLux" e pelo sensor fixado no VDT; e (b) Aquisição de dados pelos sensores fixos (Eh, DGP, grau de abertura dos painéis e status de iluminação)}

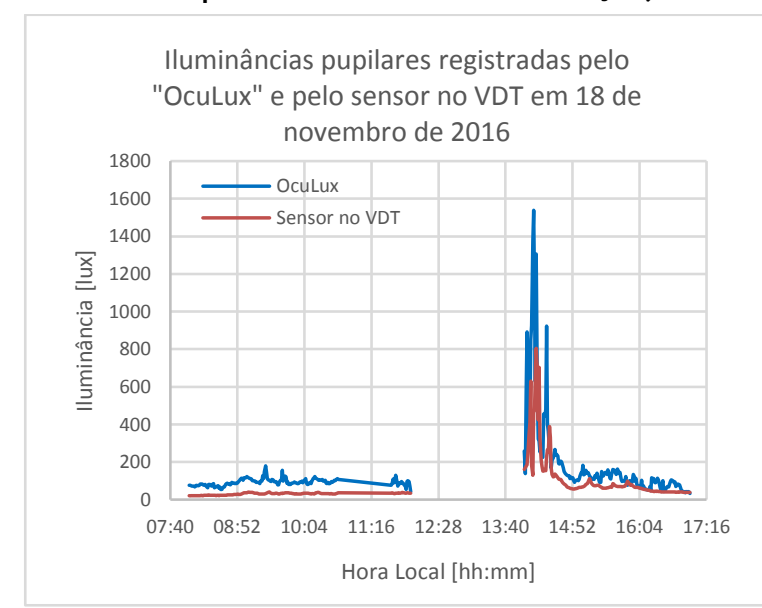

(a)

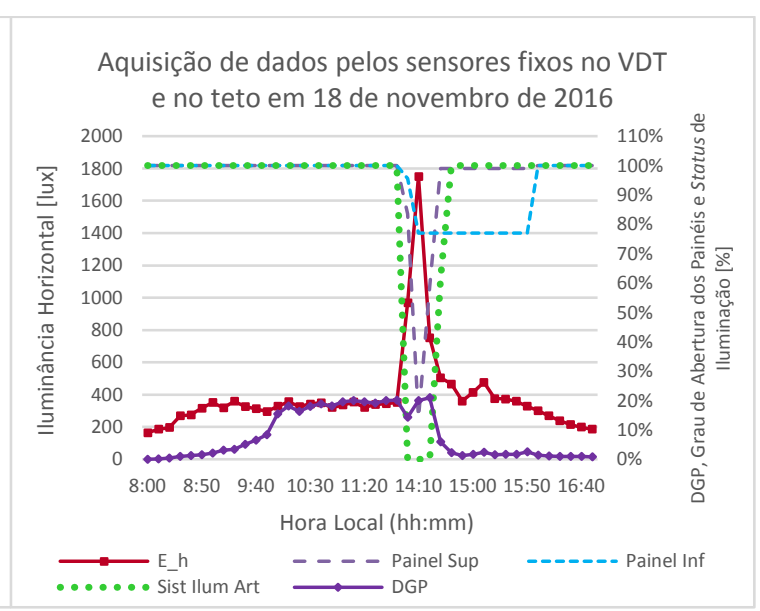

(b) 
Dados coletados para os dias com céu parcialmente encoberto apontaram um aumento nos níveis de iluminância pupilar registrados no período entre 10h15-10h45 e 14h-14h30, como pôde ser verificado no dia 28 de novembro. Todavia, para ambos os períodos mencionados os registros do "OcuLux" foram superiores aos do sensor fixo no VDT. Isso ocorreu porque ao fixar o sensor no VDT, seu o ângulo frente à janela é inferior ao ângulo para o campo visual humano (Figura 13a).

Ainda que o "OcuLux" tenha apresentado registros superiores aos do sensor fixo no VDT, pode-se observar que o dispositivo vestível responde de modo condizente às variações luminosas no ambiente analisado. Tal fato pode ser ratificado através da Figura 13b.

Como a $\mathrm{E}_{\mathrm{h}}$ se manteve inferior ao estabelecido pelo controlador, o sistema de iluminação artificial esteve acionado até às 9h20. Após esse horário, com um aumento nos índices de $E_{\mathrm{h}}$, o painel inferior foi ativado para cobrir $20 \%$ da janela convencional. Nesse mesmo período o DGP apresentou registros entre $11 \%$ e $20 \%$ e o painel superior passou a cobrir todo o ADS para minimizar as condições de ofuscamento. A atuação dos painéis contribuiu para a diminuição da $E_{\mathrm{h}}$ e do DGP. No início da tarde, o aumento da $\mathrm{E}_{\mathrm{h}}$ levou a um acionamento adicional do painel inferior, que passou então a cobrir cerca de $40 \%$ da janela. O aumento nos registros do DGP acarretou a ativação do painel superior. A atuação do sistema de sombreamento contribuiu para que os índices da $E_{h}$ e do DGP voltassem àqueles estabelecidos pelo controlador, garantindo o conforto visual no ambiente. O sistema de iluminação artificial se manteve desativado por intervenção do usuário (via controles manuais) até o final do experimento (Figura 13b).
O cálculo da dose diária de luz apontou que durante os dias com céu encoberto (cinza) o usuário recebeu entre 900 a 1.800 [lux.h], enquanto que nos dias com céu parcialmente encoberto (azul) tal registro variou entre 1.500 a 3.200 [lux.h] (Figura 14a). Devido ao fato do usuário ocupar a estação de trabalho posicionada com a janela à sua esquerda e a angulação que a radiação direta incide na fachada sul (Figura 7a), inicialmente era esperado que a dose de luz recebida durante a tarde fosse consideravelmente superior à da manhã. Contudo, o registro da dose de luz por período mostrou que não houve uma diferença significativa entre tais índices, exceto para o dia 29 de novembro (Figura 14b). Isso ocorreu devido à atuação do controlador que ativa automaticamente o sistema de sombreamento quando o DGP é superior a 5\%, e o sistema de iluminação artificial quando a $E_{h}$ no plano de trabalho é inferior a 200 lx.

No transcorrer do mês de novembro no Hemisfério Norte o período de disponibilidade de luz natural começa a ser reduzido a partir do outono. O período de coletas foi marcado por dias médios (com 8,59,5 horas de luz solar disponível), nos quais a luz natural pôde ser observada até 16h55. Para dias com céu encoberto (08, 18 e 21 de novembro) a dose de luz permaneceu estável pela manhã, com registros na faixa de $\sim 80$ [lux.h] a $\sim 180$ [lux.h]. No final do dia, o registro foi equivalente a $\sim 70$ [lux.h]. Nos dias com céu parcialmente encoberto (16, 24, 28 e 29 de novembro) houve um aumento na dose de luz registrada a partir das $10 \mathrm{~h}$, chegando a atingir 380 [lux.h]. No período da tarde, após as 15h a dose de luz decai de $\sim 530$ [lux.h] para $~ 90$ [lux.h] (Figura 15a).

Figura 13 - Dados para 28 de novembro de 2016 sob céu parcialmente encoberto: (a) Registro das iluminâncias pelo "OcuLux" e pelo sensor fixado no VDT; e (b) Aquisição de dados pelos sensores fixos ( $E_{h}, D G P$, grau de abertura dos painéis e status de iluminação)

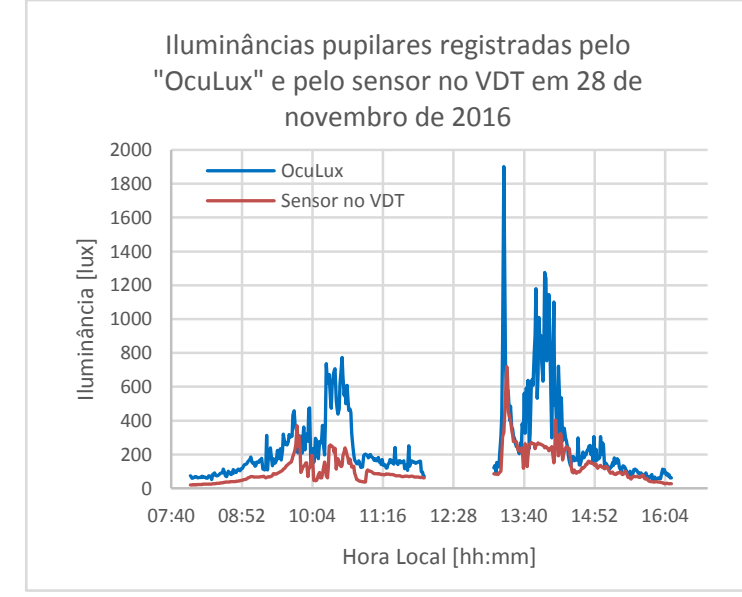

(a)

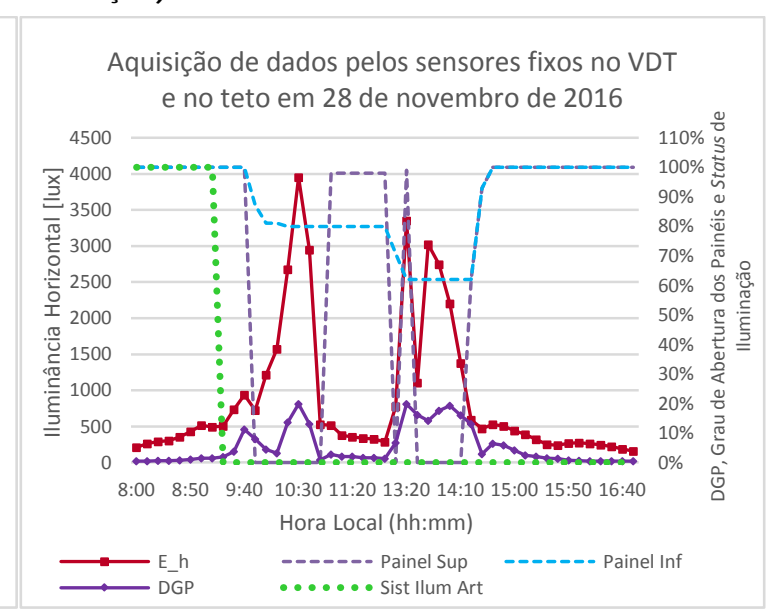

(b) 
Figura 14 - (a) Dose média de luz [lux.h] recebida pelo usuário durante o experimento; e (b) Dose de luz [lux.h] recebida por período

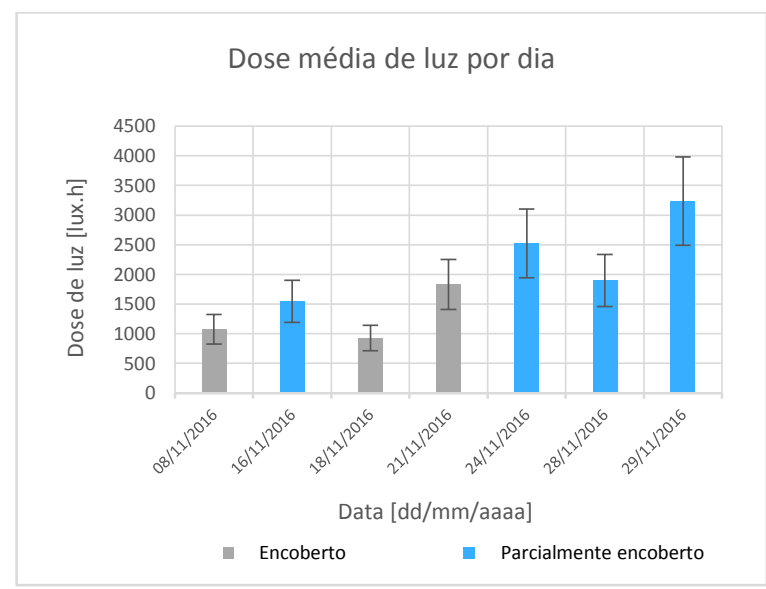

(a)

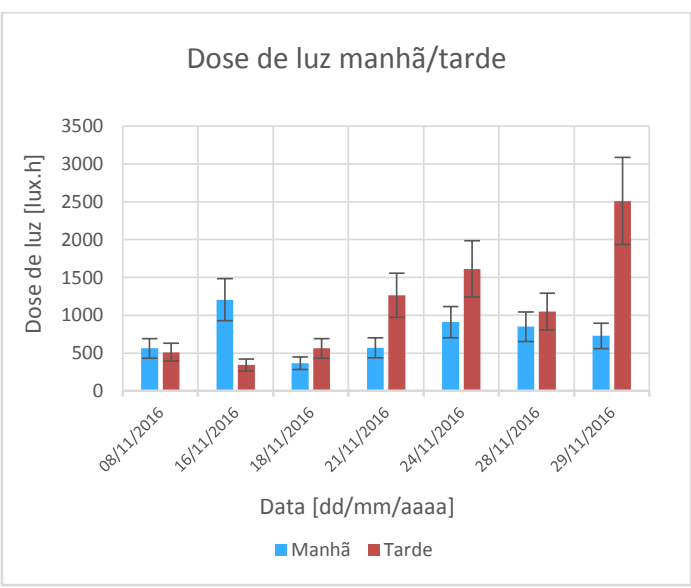

(b)

Figura 15 - (a) Dose média de luz [lux.h] recebida por hora; e (b) Duração do tempo de exposição dos diferentes níveis de iluminância como porcentagem por hora

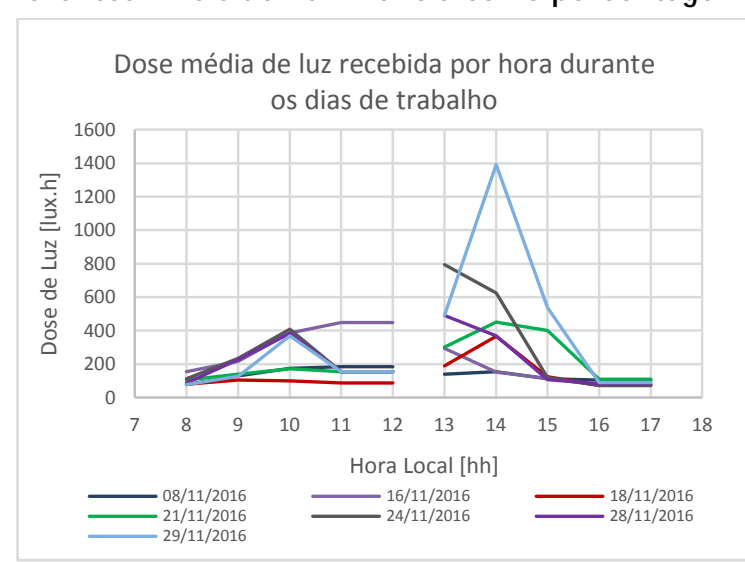

(a)

Ao verificar a distribuição dos níveis de iluminância aos quais o usuário foi exposto ao longo das horas de trabalho (Figura 15b), foi constatado que ele esteve exposto a uma iluminância pupilar entre 50150 lx durante metade do período. Registros variando entre 150-250 lx foram observados em $27 \%$ do tempo e entre $250-500 \mathrm{~lx}$ em $12 \%$ do tempo. A exposição a níveis superiores a $1.000 \mathrm{~lx}$ ocorreu em cerca de $4 \%$ do tempo.

Considerando que a $\mathrm{E}_{\mathrm{h}}$ mínima requerida pela EN 12464-1 seja de 300-500 lx no plano de trabalho e o Handbook of Industrial Engineering: Technology and Operations Management (SALVENDY, 2001) recomende que para ambientes de trabalho iluminados por luminárias suspensas a relação entre a iluminância vertical e a horizontal deve ser entre 0,3 e 0,5, uma iluminância vertical mínima igual a $150-250$ lx $(500 \times 0,3$ e 500 x 0,5) deve ser assumida. Os registros nessa faixa foram observados em $27 \%$ do tempo e mesmo que os

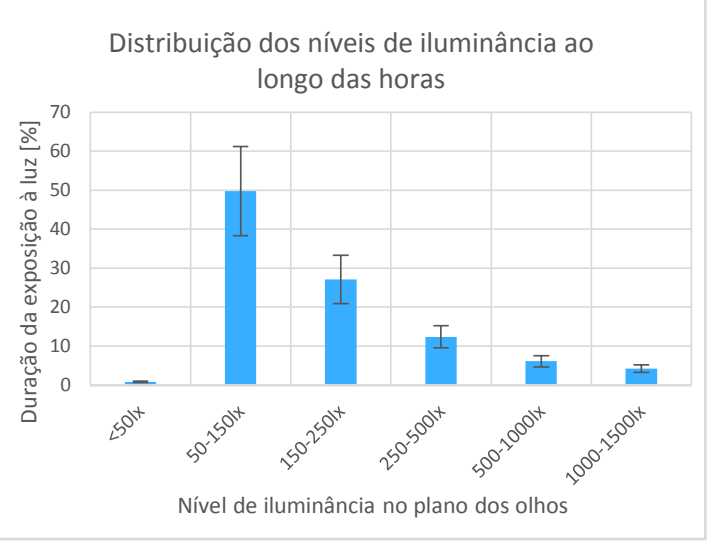

(b)

registros sejam inferiores aos requeridos pelas normas, deve-se considerar as preferências do usuário, que teve autonomia para controlar o sistema de iluminação e de sombreamento.

Visto que o controlador atua segundo os índices de DGP, $E_{h}$, posição relativa dos painéis externos motorizados e quantidade de luz solar disponível para garantir condições de conforto visual no ambiente analisado, sua programação tem interferência direta na iluminância pupilar registrada e consequentemente, na dose de luz recebida pelo usuário. Durante o estudo experimental o controlador estava programado para ativar o sistema de sombreamento para um DGP superior a 5\%. Tal índice é bastante inferior ao limite recomendado por Wienold e Christoffersen (2006) como ofuscamento imperceptível (<35\%). Desta forma, a ativação do sistema de sombreamento de acordo com os índices estabelecidos pelo controlador, bloqueou parte da 
luz solar direta incidente contribuindo para menores registros da iluminância pupilar.

\section{Considerações finais}

Mudanças no modo de realizar as tarefas de escritório decorrentes da introdução dos computadores levaram à concepção de ambientes mais dinâmicos que permitem deslocamentos e movimentos contínuos, fazendo com que todo o entorno seja considerado em pesquisas que objetivam relacionar a luz, o desempenho visual e a saúde. Nesse sentido, dispositivos de medição como o luxímetro e o luminancímetro que fornecem uma medição fixa não são suficientes.

Os dados apresentados indicam que o "OcuLux" responde de modo coerente às variações nas quantidades luminosas em um ambiente interno. A diferença entre os registros do dispositivo vestível e o sensor fixo no VDT deve-se inicialmente à localização deste último, como já foi relatado. Mesmo o equipamento apresentando faixa de medição limitada, seu uso como um dispositivo de referência na escolha da posição/orientação mais adequada para os sensores de iluminação e/ou brilho fixo podem fornecer ao sistema de automação de edifícios informações importantes sobre a situação de iluminação do ambiente, de modo a garantir conforto visual personalizado e baixo consumo de energia.

No período em que o experimento ocorreu, houve necessidade de complementar a iluminação natural da sala LE001 do LESO-PB da EPFL com a iluminação artificial para que os níveis adequados de iluminação recomendados pela EN 12464-1 (EUROPEAN..., 2011) fossem alcançados. A dose diária de luz recebida pelo usuário durante 7 dias em novembro de 2016 foi monitorada, tendo sido observado que a mesma não foi consideravelmente afetada pela altura angular do sol. Restrições de conforto visual atuam no controle do sistema de sombreamento ao limitar a penetração da luz solar no ambiente.

Estudos em ambientes não laboratoriais para determinar a radiação óptica que pode afetar a saúde humana precisam levar em consideração uma série de variáveis, incluindo o fato de que a luz natural é dinâmica. Contudo, a inexistência de parâmetros que relacionem a dose de luz recebida pela luz natural impossibilitou o aprofundamento das análises dos dados obtidos. Experimentos complementares com sujeitos de diferentes sexos, idades e grupos étnicos devem ser realizados para permitirem avaliações mais amplas sobre os dados encontrados, visto que a prioridade deve ser sempre atender aos requisitos para a realização das tarefas visuais. Investigações de longa duração em ambientes reais para diagnosticar a iluminância pupilar podem ser replicados para diferentes tipologias de ambientes e tipos de atividades.

Embora o "OcuLux" ainda necessite de alguns aperfeiçoamentos - como a substituição do microcontrolador Arduino NANO por outro mais sofisticado e um desenho mais ergonômico para o conjunto - o uso do dispositivo pode contribuir positivamente para avanços no tema, visto que hoje há um grande empenho da comunidade científica e de instituições internacionais para que parâmetros e determinantes para uma iluminação biologicamente eficaz sejam estipulados. Experimentos adicionais em andamento no LESO-PB com a utilização de ambos os sensores permitirão refinamentos no dispositivo vestível, a fim de que este se torne uma ferramenta precisa de monitoramento das condições de iluminação em ambientes internos. Desta forma, o “OcuLux” poderá vir a ser utilizado, por exemplo, como um sensor de referência na escolha pela posição e orientação ideais para sensores de iluminação.

\section{Referências}

ALTHERR, R.; GAY, J.-B. Alow Environmental Impact Anidolic Facade. Building and

Environment, v.3 7, p. 1409-1419, 2002.

ARDUINO. Versão 1.0.6. Italy: Interaction Design Institute Ivrea, [201-].

ARIES, M. Human Lighting Demands: healthy lighting in an office environment. Eindhoven, 2005. $158 \mathrm{f}$. Thesis (Doctoral Program in Building Physics and Systems) - Technische Universiteit Eindhoven, Eindhoven, 2005.

BOYCE, P. R. Human Factors in Lighting. 3. ed. USA: CRC Press, 2014.

BOYCE, P. R.; RAYNHAM, P. The SLL Lighting Handbook. London: CIBSE, 2009.

BURGESS, H. J.; EASTMAN, C. I. Early Versus Late Bedtimes Phase Shift the Human Dim Light Melatonin Rhythm Despite a Fixed Morning Lights on Time. Neuroscience Letters, v. 356, n. 2, p. 115-118, 2004.

CAJOCHEN. C. Light: an underestimated environmental factor in sleep medicine. Sleep and Biological Rhythms, v. 13, p. 111, 2015.

DIAS, M. V. et al. Toward Proper Evaluation of Light Dose in Indoor Office Environment by Frontal Lux Meter. Energy Procedia, v. 122, p. 835-840, 2017. 
DIAS, M. V. et al. Iluminação e Saúde Humana: estado da arte em dispositivos de medição de luz no nível dos olhos. Pós. Revista do Programa de Pós-Graduação em Arquitetura e Urbanismo da FAUUSP, v. 21, p. 210-227, 2015.

DEUTSCHES INSTITUT FÜR NORMUNG. DIN SPEC 67000: biologically effective illumination: design guidelines. Berlin, 2013.

EUROPEAN STANDARD. EN 12464-1: light and lighting: lighting of work places: part 1: indoor work places. Brussels, 2011.

HAMAMATSU PHOTONICS. Color Sensor. S9706. 12-bit digital output. Hamamatsu: Hamamatsu, 2016.

HUBALEK, S.; SCHIERZ, C. LichtBlick: photometrical situation and eye movements at VDU work places. In: EUROPAISCHER LICHTKONGRESS LUX EUROPA 2005: LIGHTING FOR HUMANS, Berlin, 2005. Proceedings... Berlin: Lux Europa, 2005.

HUBALEK, S.; ZOSCHG, D.; SCHIERZ, C. Ambulant Recording of Light For Vision and NonVisual Biological Effects. Lighting Research \& Technology, v. 38, n. 4, p. 314-324, 2006.

ILLUMINATING ENGINEERING SOCIETY OF NORTH AMERICA. IES TM-18-08 Light and

human health: an overview of the impact of optical radiation on visual, circadian, neuroendocrine and neurobehavioral responses. New York: IES, 2008.

JACOBS, A. Radiance Cookbook. Reino Unido: Jaloxa, 2014. Disponível em:

$<$ http://www.jaloxa.eu/resources/radiance/docume ntation/docs/radiance_cookbook.pdf $>$. Acesso em: 29 out. 2016.

JERNIGAN, R. C. Light Studies Focus on

Circadian Rhythms. Massachusetts:

BioPhotonics, 2009. Disponível em:

$<$ www.photonics.com/Article.aspx?AID=38995 $>$. Acesso em: 08 mar. 2013.

KAIDA, K. et al. Indoor Exposure to Natural Bright Light Prevents Afternoon Sleepiness. Sleep, v. 29, n. 4, 2006.

LAUSANNE. Lausanne in Short. Lausanne:

Disponível em:

$<$ http://www.lausanne.ch/en/lausanne-en-

bref/lausanne-un-portrait.html> . Acesso em: 26 mar. 2017.

LIGHTING RESEARCH CENTER. The

Dimesimeter. 2013. Disponível em:

<http://www.lrc.rpi.edu/programs/lightHealth/proj ects/Dimesimeter.asp>. Acesso em: 18 mar. 2013.
LINHART, F. Energetic, Visual and Non-Visual Aspects of Office lighting. Lausanne, 2010. 295 f. Thesis (Doctoral Program in Environment) - École Polytechnique Fédérale de Lausanne, Lausanne, 2010.

MATLAB. Versão R2016b. Massachusetts: The MathWorks, 2016.

MOTAMED, A.; DESCHAMPS, L.;

SCARTEZZINI, J.-L. On-Site Monitoring and Subjective Comfort Assessment of a Sun Shadings and Electric Lighting Controller Based on Novel High Dynamic Range vision sensors. Energy and Buildings, v. 149, p. 58-72, 2017.

MOTAMED, A.; DESCHAMPS, L.;

SCARTEZZINI, J.-L. Toward an Integrated Platform For Energy Efficient Lighting Control of Non-Residential Buildings. In: SUSTAINABLE BUILT ENVIRONMENT REGIONAL CONFERENCE: EXPANDING BOUNDARIES: SYSTEMS THINKING IN THE BUILT ENVIRONMENT, Zurich, 2016. Proceedings... Zurich: SBE, 2016.

MÜNCH, M. et al. Effects of Prior Light Exposure on Early Evening Performance, Subjective Sleepiness, and Hormonal Secretion. Behavioral Neuroscience, v. 126, n. 1, p. 196-203, 2012.

PHILIPS RESPIRONICS. Helpful Hints For Filing: actigraphy studies. 2011. Disponível em: $<$ http://incenter.medical.philips.com/doclib/enc/98 38742/ActigraphyReimbursementHelpfulHints.pdf \%3ffunc\%3ddoc.Fetch\%26nodeid\%3d9838742>. Acesso em: 30 dez. 2012.

SALVENDY, G. Handbook of Industrial Engineering: technology and operations management. 3. ed. New Jersey: John Wiley \& Sons, 2001.

SOLAR ENERGY AND BUILDING PHYSICS LABORATORY LESO-PB. Anidolic Systems: façade. 2 fotografias, color. Lausanne: EPFL. Disponível em: < https://leso.epfl.ch/page-43417en.html>. Acesso em: 16 nov. 2016.

VANDAHL, C. et al. Light and Health in Factory Work Places. In: CIE 2011, South Africa, 2011. Proceedings... South Africa: CIE, 2011.

WIENOLD, J.; CHRISTOFFERSEN, J. Evaluation Methods and Development of a New Glare Prediction Model For Daylight Environments With the Use of CCD Cameras. Energy and Buildings, v. 38, p. 743-757, 2006.

\section{Agradecimentos}

Os autores agradecem o suporte oferecido pela Fundação de Amparo à Pesquisa do Estado de São 
Paulo - FAPESP (Processos n ${ }^{\circ}$ 2012/08887-1 e $n^{\circ}$ 2015/23213-5) bem como à Commission for Innovation and Technology (CTI) da Confederação
Suíça através do Swiss Competence Center for Energy Research on Future Energy Efficient Buildings and Districts (SCCER FEED\&B).

\section{Maíra Vieira Dias}

Departamento de Arquitetura e Construção, Faculdade de Engenharia Civil, Arquitetura e Urbanismo | Universidade Estadual de Campinas | Rua Albert Einstein, 951, Barão Geraldo | Campinas - SP - Brasil | CEP 13083-889 | Tel.: (19) 3521-2383 | E-mail:

mairavd@yahoo.com.br

\section{Ali Motamed}

Solar Energy and Building Physics Laboratory | École Polytechnique Fédérale de Lausanne | EPFL-ENAC-IIC-LESO-PB | CH-1015 | Lausanne - Switzerland | Tel.: +44 (21) 693-6265 | E-mail: ali.motamed@epfl.ch

\section{Paulo Sergio Scarazzato}

Departamento de Arquitetura e Construção, Faculdade de Engenharia Civil, Arquitetura e Urbanismo | Universidade Estadual de Campinas | E-mail: paulosca@fec.unicamp.br

\section{Jean-Louis Scartezzini}

Solar Energy and Building Physics Laboratory | École Polytechnique Fédérale de Lausanne | Tel.: +44 (21) 693-4545 | E-mail: jeanlouis.scartezzini@epfl.ch

\section{Revista Ambiente Construído}

Associação Nacional de Tecnologia do Ambiente Construído

Av. Osvaldo Aranha, 99 - 3o andar, Centro

Porto Alegre - RS - Brasil

$$
\text { CEP } 90035-190
$$

Telefone: +55 (51) 3308-4084

Fax: +55 (51) 3308-4054

www. seer. ufrgs. br/ ambienteconstruido

E-mail: ambienteconstruido@ufrgs.br 